PROCEEDINGS OF THE

AMERICAN MATHEMATICAL SOCIETY

Volume 139, Number 11, November 2011, Pages 4105-4111

S 0002-9939(2011)10810-4

Article electronically published on March 17, 2011

\title{
GROWTH AND ISOPERIMETRIC PROFILE OF PLANAR GRAPHS
}

\author{
ITAI BENJAMINI AND PANOS PAPASOGLU
}

(Communicated by Jianguo Cao)

\begin{abstract}
Let $\Gamma$ be a planar graph such that the volume function of $\Gamma$ satisfies $V(2 n) \leq C V(n)$ for some constant $C>0$. Then for every vertex $v$ of $\Gamma$ and $n \in \mathbb{N}$, there is a domain $\Omega$ such that $B(v, n) \subset \Omega, \partial \Omega \subset B(v, 6 n)$ and $|\partial \Omega| \precsim n$.
\end{abstract}

\section{INTRODUCTION}

It is known that a planar path metric space satisfying a subquadratic isoperimetric inequality must satisfy a linear isoperimetric inequality. See e.g. 6.

Recently, motivated by physics, quantum gravity in particular (see [1]), there is an emerging interest in random planar geometries of several types, which sometimes are modeled by planar triangulations. These can admit strange volume growth functions for planar triangulations; e.g., balls of radius $r$ have volume $r^{4}$. What does a surface of polynomial volume growth larger than quadratic around any vertex look like? In this paper we comment that although growth can be big, the volume is access via big fractal folds. That is, such metric spaces still must have small cutsets at all scales. For such graphs we establish a stronger isoperimetric result, stronger than what follows from the dichotomy mentioned above.

The isoperimetric profile is linked to heat kernel estimates; see e.g. 9] for a very recent monograph on the topic. We hope our result might be useful in proving anomalous subdiffusion rates for simple random walks on such graphs, which is conjectured, and might help in identifying the conformal type of such surfaces. More on that at the end.

For clarity we chose to work in the discrete setup of graphs; similar results hold for Riemannian metrics on the plane and other planar metric spaces.

Let $\Gamma$ be a locally finite graph. If $a$ is a vertex of $\Gamma$ we denote by $B(a, n)$ the ball of radius $n$ centered at $a$. If $B$ is a graph, we denote by $|B|$ the number of vertices of $B$.

Define the growth function of $\Gamma$ at $a$ by

$$
V(a, n)=|B(a, n)| .
$$

A graph $\Gamma$ is doubling if there is a constant $C>0$ such that for all $a, b \in \operatorname{Vert}(\Gamma)$ and $n \in \mathbb{N}, V(a, 2 n) \leq C V(b, n)$. We say then that $C$ is a doubling constant for $\Gamma$.

If $\Gamma$ is a doubling graph, then the degree of the vertices is uniformly bounded.

Received by the editors April 24, 2010 and, in revised form, September 24, 2010

2010 Mathematics Subject Classification. Primary 53C20, 53C23, 05C10.

(C)2011 American Mathematical Society Reverts to public domain 28 years from publication 
Note that for any $d \geq 1$, there are planar graphs with the doubling property for which, for any $a$ and $n, V(a, n)$ is of order $n^{d}$; see e.g. the last section of [5].

We say that a graph $\Gamma$ corresponds to a tessellation of $\mathbb{R}^{2}$ if there is a $k \in \mathbb{N}$ such that all components of $\mathbb{R}^{2}-\Gamma$ are bounded regions with at most $k$ sides.

Definition. Let $(X, d)$ be a metric space. An $\epsilon$-net $N$ of $X$ is a set such that $d\left(v_{1}, v_{2}\right)>\epsilon$ for all $v_{1}, v_{2} \in N$ and $N$ is a maximal set with this property.

We remark that if $N$ is an $\epsilon$-net of $X$, then $X$ is contained in the $\epsilon$-neighborhood of $N$.

Definition. Let $\Omega$ be a subgraph of a graph $\Gamma$. Then we denote by $\partial \Omega$ the set of all vertices of $\Gamma-\Omega$ which have a neighbor in $\Omega$.

Our main result is the following:

Theorem 2.1. Let $\Gamma$ be a doubling planar graph. Then there is a constant $\alpha$ so that for every vertex $v$ of $\Gamma$ and $n \in \mathbb{N}$ there is a finite domain $\Omega$ such that $B(v, n) \subset \Omega$, $\partial \Omega \subset B(v, 6 n)$ and $|\partial \Omega| \leq \alpha n$. $\alpha$ depends only on the doubling constant.

Krikun [10] has shown a similar theorem for the uniform infinite planar triangulation (UIPT) introduced in 3 . The volume doubling property does not hold for the UIPT; still an asymptotic version should hold: for any vertex $v$, for large enough $n, B(v, 2 n)$ contains order 1 disjoint balls of radius $n / 2$ a.s., and the proof below will adapt to give Krikun's result. The asymptotic volume growth of balls in the UIPT is of order $n^{4}$, up to polylogs (see [2]), and thus a weaker result with a polylog correction follows from our result. For the uniform infinite planar quadrangulation a sharp volume growth estimate is known which implies the asymptotic volume doubling [11, 7]. The same asymptotic result is conjectured for the UIPT. For a detailed study of the geometry of the uniform infinite planar quadrangulation and a matching lower bound on the small cuts, see [7.

Our result shows that the volume and the isoperimetric profile function are related for planar graphs. We define here the isoperimetric profile function of a graph in a similar way as for Riemannian manifolds:

Definition. Let $\Gamma$ be a locally finite graph and let $V(n)$ be the volume function of $\Gamma$. Then the isoperimetric profile function of $\Gamma, I_{\Gamma}: \mathbb{N} \rightarrow \mathbb{N}$, is defined by

$$
I_{\Gamma}(n)=\inf _{\Omega}\{|\partial \Omega|: \Omega \subset \Gamma,|\Omega| \leq n\},
$$

where $\Omega$ ranges over all subgraphs of $\Gamma$.

From Theorem 2.1 we obtain the following:

Corollary 1.1. Let $\Gamma$ be a doubling planar graph with volume function $V(n)$ and isoperimetric profile function $I_{\Gamma}(n)$.

Let $\varphi(n)=\inf \{k: V(k) \geq n\}$. Then there is a constant $\alpha$ such that

$$
I_{\Gamma}(n) \leq \alpha \varphi(n) \text { for all } n \in \mathbb{N} .
$$

The same result holds for Riemannian metrics on the plane or on the plane with holes (again assuming the doubling property holds for the metric), and our proof extends to this setting as well (see [12] for more information on isoperimetric profiles of planes and planes with holes). 
1.1. Idea of the proof. Here is a sketch of the proof of Theorem 2.1. Let $v$ be any vertex of $\Gamma$. Consider the balls $B(v, n), B(v, 3 n)$. Let $N$ be an $n$-net of $\partial B(v, 3 n)$. For each vertex $w$ of $N$ consider $B(w, n / 2)$. Note that all such balls are disjoint since $N$ is an $n$-net. Also all these balls are contained in $B(v, 4 n)$. So, by the doubling property, we can have only boundedly many such balls; that is, $|N| \leq \beta$, where $\beta$ does not depend on $n$. Consider now the balls $B(w, 2 n)$ for all $w \in N$. $\partial B(v, 3 n)$ is contained in the union of these balls. Construct a closed curve that 'blocks' $v$ from infinity as follows: if $w_{1}, w_{2} \in N$ are such that $d\left(w_{1}, w_{2}\right) \leq 2 n$, then we join them by a geodesic. So replace $\partial B(v, 3 n)$ by the 'polygonal line' that we define using vertices in $N$. This 'polygonal line' blocks $v$ from infinity and has length at most $2 n \beta$. In the next section we make precise this idea. There are some technical issues to take care of; for example, $\partial B(v, 3 n)$ might not be connected (and could even have 'large gaps'), and the geodesic segments have to be chosen carefully. In particular the constants obtained will be slightly different from the ones in this sketch.

\section{Growth AND PROFILE}

Definition. Let $\Gamma$ be an infinite planar locally finite graph. We say that an embedding of $\Gamma$ in the plane is tame if for any bounded subset $A$ of the plane, $\Gamma-A$ has at least one connected component of infinite diameter. It is easy to see that any infinite planar locally finite graph admits a tame embedding to $\mathbb{R}^{2}$.

We now view $\Gamma$ as embedded in the plane by a tame embedding. Let $B=B(v, r)$ be a ball of $\Gamma$ and let $U$ be the unbounded connected component of $\mathbb{R}^{2}-B$. Define the contour of $B(v, r)$ to be the graph $B \cap \bar{U}$. Note that the contour of $B(v, r)$ is connected.

Recall some basic facts about winding numbers (see e.g. 8], ch. 3, for a definition and basic properties of winding numbers).

Let $\gamma$ be a closed curve on the plane and $v$ be a point that does not lie on $\gamma$. If the winding number $W(\gamma, v)$ is nonzero, then $v$ lies in a bounded component of $\mathbb{R}^{2}-\gamma$. If $\gamma=\gamma_{1} \cup \gamma_{2}$, where $\gamma_{1}, \gamma_{2}$ are paths with the same endpoints $a, b$ and if $\delta$ is another path with endpoints $a, b$, then

$$
W(\gamma, v)=W\left(\gamma_{1} \cup \delta, v\right)+W\left(\gamma_{2} \cup \delta, v\right) .
$$

If $\gamma$ is a closed curve, then $W(\gamma, v) \in \mathbb{Z}$. Here it will be convenient to consider the winding number modulo 2 , so in what follows by winding number of $\gamma$ around $v$ we mean $W(\gamma, v) \bmod 2$.

Theorem 2.1. Let $\Gamma$ be a doubling planar graph. Then there is a constant $\alpha$ so that for every vertex $v$ of $\Gamma$ there is a finite domain $\Omega$ such that $B(v, n) \subset \Omega$, $\partial \Omega \subset B(v, 6 n)$ and $|\partial \Omega| \leq \alpha n$.

Proof. Consider a tame embedding of $\Gamma$ in the plane. Let $F$ be the contour of $B(v, 4 n)$ with respect to this embedding. Clearly $F$ is a connected subset of the plane. 'Parametrise' $F$ by a map $f: S \rightarrow F$, where $S$ is a graph homeomorphic to the circle, $f$ sends edges to edges and every vertex $w$ such that $d(w, v)=4 n$ has a unique pre-image $f^{-1}(w)$. Note that $F$ is not necessarily a simple closed curve; for example, consider the case that $\Gamma$ is a tree. Denote by $B^{\prime}$ the set of all vertices $w$ in $\Gamma$ such that $d(w, v)=4 n$. 
It is possible that $v \in F=i m f$. As this creates some technical problems, we modify $f$ slightly to avoid this, so that the curve $f(S)$ goes around $v$. For example we may do this as follows: Cyclically order the edges adjacent to $v$, as $e_{1}=\left[v, v_{1}\right], \ldots, e_{k}=\left[v, v_{k}\right]$ and introduce new edges $e_{1}^{\prime}, \ldots, e_{k}^{\prime}$ so that the edge $e_{i}$ 's joins $v_{i}, v_{i+1}\left(i \in \mathbb{Z}_{k}\right)$. One may assume that the $e_{i}$ 's do not intersect the interior of any edge of $\Gamma$ and that the only edges contained in the interior of $e_{1}^{\prime} \cup \ldots \cup e_{k}^{\prime}$ are $e_{1}, \ldots, e_{k}$.

If for two successive edges $g_{1}, g_{2}$ of $S$, we have $f\left(g_{1}\right)=e_{i}, f\left(g_{2}\right)=e_{i+1}$, then modify $f$ so that $f\left(g_{1} \cup g_{2}\right)=e_{i}^{\prime}$.

After this modification the winding number of $f(S)$ around $v$ is defined and it is equal to 1 . If $S_{1}$ is a subarc of $S$, define the weight of $S_{1}$ to be $w\left(S_{1}\right)=\left|f\left(S_{1}\right) \cap B^{\prime}\right|$.

Note that if $a, b \in B^{\prime}$, then there are two subarcs $S_{1}, S_{2}$ of $S$ such that $S=$ $S_{1} \cup S_{2}$ and $f\left(S_{1}\right), f\left(S_{2}\right)$ are paths with endpoints $a, b$. If we further assume that $d(a, b)<4 n$ and $\gamma$ is a geodesic in $\Gamma$ joining $a, b$, then $v \notin \gamma$, so one of the closed curves $f\left(S_{1}\right) \cup \gamma$ and $f\left(S_{2}\right) \cup \gamma$ has winding number 1 around $v$, while the other has winding number 0 around $v$.

If for any two vertices $a, b$ of $B^{\prime}, d(a, b)>2 n+1$, then the balls $B(a, n)$, where $a \in B^{\prime}$, are disjoint. So by the doubling property $\left|B^{\prime}\right| \leq C^{3}$, where $C$ is the doubling constant of $\Gamma$. Therefore in this case one can take $\Omega=B(v, 4 n)$.

Otherwise pick $a, b$ in $B^{\prime}$ with the following two properties:

1) $d(a, b) \leq 2 n+1$.

2) There is a geodesic $\gamma$ in $\Gamma$ joining $a, b$ so that for the subarc $S_{1} \subset S$ for which the closed curve $f\left(S_{1}\right) \cup \gamma$ has winding number 1 around $v, w\left(S_{1}\right)$ is the minimum possible.

If $f\left(S_{1}\right) \cap B^{\prime}=\{a, b\}$ we may take $\Omega$ to be the component of $\Gamma-\gamma$ containing $v$, and the theorem is proved.

Otherwise, observe that $f\left(S_{1}\right) \cup \gamma$ separates $v$ from infinity. We now explain how to replace $f\left(S_{1}\right)$ by a curve of length linear in $n$.

Consider an $n$-net $N$ of the set $B^{\prime} \cap f\left(S_{1}\right)$. Observe that if $x, y \in N$, the balls $B(x, n / 2), B(y, n / 2)$ are disjoint and are contained in $B(v, 9 n / 2)$. It follows that $|N|<C^{4}$.

Define a graph $\Delta$ with set of vertices equal to $N \cup\{a, b\}$. Join two vertices $x, y$ by an edge if $d(x, y) \leq 2 n+1$.

We consider first the case that $\Delta$ is connected. Then there is a simple path in $\Delta$ joining $a, b$. This simple path corresponds to a path $\delta$ in $\Gamma$. We obtain $\delta$ by replacing edges of the path in $\Delta$ by geodesic paths of length $\leq 2 n+1$ in $\Gamma$. It follows that

$$
\text { length }(\delta) \leq(2 n+1) C^{4} .
$$

Consider now the closed path

$$
p=\gamma \cup \delta .
$$

We claim that the winding number of $p$ around $v$ is 1 . Indeed $\delta=\delta_{1} \cup \ldots \cup$ $\delta_{k}$, where $\delta_{i}=\left[a_{i}, a_{i+1}\right]$ are geodesic paths of $\Gamma$ corresponding to edges of $\Delta$ as explained above and $a=a_{1}, b=a_{k+1}$. Clearly there are $a_{1}^{\prime}, \ldots, a_{k+1}^{\prime} \in S_{1}$ such that $f\left(a_{i}^{\prime}\right)=a_{i}$ and

$$
\bigcup_{i=1}^{k}\left(a_{i}^{\prime}, a_{i+1}^{\prime}\right)=S_{1} .
$$


The curve

$$
f\left(a_{i}^{\prime}, a_{i+1}^{\prime}\right) \cup \delta_{i}
$$

is closed and has winding number 0 around $v$. This follows from the definition of $\gamma$ and the fact that the weight of $\left(a_{i}^{\prime}, a_{i+1}^{\prime}\right)$ is smaller than the weight of $S_{1}$. By the additivity property of winding numbers we see that by replacing successively $f\left(a_{i}^{\prime}, a_{i+1}^{\prime}\right)$ by $\delta_{i}$ in $f\left(S_{1}\right) \cup \gamma$ the winding number around $v$ remains the same. So the winding number of $p$ around $v$ is 1 , and the claim is proved.

Let $\Omega$ be the component of $\Gamma-p$ containing $v$. $\Omega$ clearly has all the properties required by the theorem.

Let us return now to the proof of the general case, so we do not assume anymore that $\Delta$ is connected.

Let $\Delta_{1}$ be the connected component of $\Delta$ containing $a$. Let $V_{1}$ be the vertex set of $\Delta_{1}$.

Let us denote by $a^{\prime}, b^{\prime}$ the endpoints of $S_{1}$, so $f\left(a^{\prime}\right)=a, f\left(b^{\prime}\right)=b$.

Let $a_{2}^{\prime} \in S_{1}$ be such that

$$
d\left(f\left(a_{2}^{\prime}\right), V_{1}\right) \leq n
$$

and $f\left[a^{\prime}, a_{2}^{\prime}\right]$ contains all vertices of $f\left(S_{1}\right)$ that are at a distance $\leq n$ from $V_{1}$. Join $f\left(a_{2}^{\prime}\right)$ to a vertex of $\Delta_{1}$ by a geodesic $\gamma_{1}$ of length $\leq n$. If $d_{1}=\left|V_{1}\right|$, then, as before, we see that there is a path $\delta_{1}$ joining $a$ to $f\left(a_{2}^{\prime}\right)$ such that

$$
\text { length }\left(\delta_{1}\right) \leq d_{1}(2 n+1)
$$

and the winding number of $\gamma \cup \delta_{1} \cup f\left[a_{2}^{\prime}, b^{\prime}\right]$ around $v$ is 1 .

Let $b_{2}^{\prime}$ be the first vertex of $S_{1}$ following $a_{2}^{\prime}$ such that $d\left(f\left(b_{2}^{\prime}\right), v\right)=4 n$. Consider an $n$-net $N_{1}$ of the set $B^{\prime} \cap f\left(a_{2}^{\prime}, b^{\prime}\right)$. Clearly $N_{1} \cup V_{1}$ is an $n$-net of the set $B^{\prime} \cap f\left(S_{1}\right)$, so $\left|N_{1} \cup V_{1}\right| \leq C^{4}$. As before define a graph with vertex set $\left\{f\left(b_{2}^{\prime}\right)\right\} \cup N_{1}$ and consider the connected component, say $\Delta_{2}$, of this graph which contains $f\left(b_{2}^{\prime}\right)$. If $V_{2}$ is the vertex set of $\Delta_{2}$, let $a_{3}^{\prime} \in S_{1}$ be such that

$$
d\left(f\left(a_{3}^{\prime}\right), V_{2}\right) \leq n
$$

and $f\left[b_{2}^{\prime}, a_{3}^{\prime}\right]$ contains all vertices of $f\left(S_{1}\right)$ that are at distance $\leq n$ from $V_{2}$. Join $f\left(a_{3}^{\prime}\right)$ to a vertex of $\Delta_{1}$ by a geodesic $\gamma_{2}$ of length $\leq n$. If $d_{2}=\left|V_{2}\right|$, then, as before, we see that there is a path $\delta_{2}$ joining $f\left(b_{2}^{\prime}\right)$ to $f\left(a_{3}^{\prime}\right)$ such that

$$
\text { length }\left(\delta_{2}\right) \leq d_{2}(2 n+1)
$$

and the winding number of

$$
\gamma \cup \delta_{1} \cup f\left[a_{2}^{\prime}, b_{2}^{\prime}\right] \cup \delta_{2} \cup f\left[a_{3}^{\prime}, b^{\prime}\right]
$$

around $v$ is 1 .

We continue in the same way. Consider an $n$-net $N_{2}$ of $f\left[a_{3}^{\prime}, b^{\prime}\right] \cap B^{\prime}$ and define similarly a graph $\Delta_{3}$ and a path $\delta_{3}$. Clearly this procedure terminates and eventually we produce paths $\delta_{1}, \ldots, \delta_{k}$ such that:

1) The winding number of

$$
\gamma \cup \delta_{1} \cup f\left[a_{2}^{\prime}, b_{2}^{\prime}\right] \cup \ldots \cup f\left[a_{k}^{\prime}, b_{k}^{\prime}\right] \cup \delta_{k}
$$

around $v$ is 1 .

2) $f\left(a_{i}^{\prime}, b_{i}^{\prime}\right) \cap B^{\prime}=\emptyset$ for all $i=2, \ldots, k$.

3) $\sum_{i=1}^{k}$ length $\left(\delta_{i}\right) \leq C^{4}(2 n+1)$.

4) The paths $\gamma, \delta_{1}, \delta_{2}, \ldots, \delta_{k}$ are contained in $B(v, 6 n)-B(v, n)$. 
Let $\Omega$ be the connected component of $\Gamma-\left(\gamma \cup \delta_{1} \cup \ldots \cup \delta_{k}\right)$ containing $v$. By the definition of $\Omega, B(v, n) \subset \Omega$. By property 3 above, $|\partial \Omega| \leq\left(C^{4}+1\right)(2 n+1)$.

Remarks. 1. If $\Gamma$ corresponds to a tessellation, then the proof of the theorem shows that there is a simple closed curve of length $\sim n$ in $B(v, 6 n)$ that separates $B(v, n)$ from infinity. In the random context this has been shown by Krikun ([10, [1]) for triangulations and quadrangulations for balls around the 'root vertex'. Our proof applies regardless of the 'shape' of the regions of the tessellation and with uniform bounds for any vertex $v$ of the graph.

2. Given any $\epsilon>0$ one may easily adapt the proof of the theorem to produce regions $\Omega$ with the same properties such that

$$
\partial \Omega \subset B(v,(1+\epsilon) n)
$$

for $n$ sufficiently large. Of course in this case one obtains $|\partial \Omega| \leq C(\epsilon) n$ and $C(\epsilon) \rightarrow$ $\infty$ as $\epsilon \rightarrow 0$.

\section{Further COMments}

A graph $G$ admits unform volume growth $f(n)$ if there are $0<c<C<\infty$ so that for all $n$, any ball of radius $n$ in $G$ satisfies

$$
c f(n)<|B(v, n)|<C f(n) .
$$

For planar graphs admitting arbitrarily large uniform polynomial growth, see e.g. 5. It is conjectured that planar graphs of uniform polynomial growth are recurrent for the simple random walk. It is also conjectured 3 that the UIPT is recurrent; see also [5]. By the Nash-Williams sufficient condition for recurrence, it is enough to find infinitely many disjoint cutsets $\left\{C_{i}\right\}$ separating the root from infinity so that $\sum\left|C_{i}\right|^{-1}=\infty$. The theorem above is a step in that direction. Still we do not know if planar graphs of uniform polynomial growth admit such cutsets. Maybe not.

Assume $G$ is a planar graph of uniform polynomial growth $n^{d}, d>2$; by the theorem above, $G$ admits bottlenecks. This suggests the conjecture that a simple random walk on $G$ will be subdiffusive, as it will spend a lot of time in domains with small boundary before exiting. That is, the expected distance to the root by time $t$ is bounded by $t^{\alpha}$ for $\alpha<1 / 2$. Does $\alpha=d^{-1}$ ? Subdiffusivity was recently established for the UIPT by Nicolas Curien and the first author.

What about a high-dimensional generalization. A $d$-sphere packing is a collection of $d$-dimensional balls with disjoint interiors. Associated to the packing is an unoriented graph $G=(V, E)$ called the d-tangency graph, where vertices corresponds to the $d$-balls and edges are between any two tangent balls; see 4. Is it the case that for any $d$, a $d$-tangency graph with the doubling property admits cutsets outside a ball of radius $n$ of size $n^{d-1}$ ?

Let $G$ be a planar triangulation which is doubling and further assume that all balls have growth $r^{d}, d>2$ up to a multiplicative constant. Is there such a $G$ for which all complements of balls are connected for all balls? Or as in the UIPT, do the complements of some balls admit several connected components, some of size proportional to the ball? 


\section{REFERENCES}

[1] J. Ambjørn, B. Durhuus and T. Jonsson, Quantum geometry. A statistical field theory approach. Cambridge University Press, Cambridge (1997). MR1465433 (98i:82001)

[2] O. Angel, Growth and percolation on the uniform infinite planar triangulation, Geometric and Functional Analysis 13, 935-974 (2003). MR2024412 (2005b:60015)

[3] O. Angel and O. Schramm, Uniform infinite planar triangulations, Comm. Math. Phys. 241, 191-213 (2003). MR2013797 (2005b:60021)

[4] I. Benjamini and N. Curien, On limits of graphs sphere packed in Euclidean space and applications, European J. Combinatorics, to appear (2010). http://arxiv.org/abs/0907.2609

[5] I. Benjamini and O. Schramm, Recurrence of distributional limits of finite planar graphs, Electron. J. Probab. 6, 13 pp. (2001). MR.1873300(2002m:82025)

[6] B. Bowditch, A short proof that a subquadratic isoperimetric inequality implies a linear one, Michigan Math. J. 42 (1991) no. 1, pp. 103-107. MR1322192(96b:20046)

[7] N. Curien, L. Menard and G. Miermont, The uniform infinite planar quadrangulation seen from infinity. In preparation.

[8] W. Fulton, Algebraic Topology, Springer, 1995. MR1343250 (97b:55001)

[9] A. Grigor'yan, Heat Kernel and Analysis on Manifolds. American Mathematical Society (2009). MR2569498

[10] M. Krikun, Uniform infinite planar triangulation and related time-reversed critical branching process, arXiv:math/0311127

[11] M. Krikun, Local structure of random quadrangulations, arXiv:math/0512304

[12] P. Papasoglu, Cheeger constants of surfaces and isoperimetric inequalities, Trans. Amer. Math. Soc. 361 (2009), 5139-5162. MR2515806

Department of Mathematics, Weizmann Institute, Rehovot, 76100, Israel

E-mail address: itai.benjamini@weizmann.ac.il

Mathematical Institute, University of Oxford, 24-29 St Giles', Oxford, OX1 3LB, United KingDOM

E-mail address: papazoglou@maths.ox.ac.uk 